\title{
GENERATORS FOR LOCALLY COMPACT GROUPS
}

\author{
by JOAN CLEARY and SIDNEY A. MORRIS
}

(Received 10th December 1991, revised 18th December 1992)

\begin{abstract}
It is proved that if $G$ is any compact connected Hausdorff group with weight $w(G) \leqq c, \mathbb{R}$ is the topological group of all real numbers and $n$ is a positive integer, then the topological group $G \times \mathbb{R}^{n}$ can be topologically generated by $n+1$ elements, and no fewer elements will suffice.
\end{abstract}

1980 Mathematics subject classification: 22D05

\section{Introduction}

A locally compact group is said to be monothetic if it can be topologically generated by one element. Within the class of compact connected Hausdorff abelian groups, the monothetic groups are those which have weight at most $c$, the cardinality of the continuum [4, §25.14]. Hofmann and Morris [5, Theorem 4.13] have shown that a compact connected Hausdorff group $G$ is topologically generated by two elements if and only if $G$ has weight $w(G) \leqq c$.

Kronecker's approximation theorem (for example, [6, Theorem 28]) can be interpreted as saying that the number of elements required to topologically generate $\mathbb{R}^{n}$ is exactly $n+1$, where $n$ is any non-negative integer.

In this paper we show that if $G$ is a compact connected Hausdorff group with weight $w(G) \leqq c$, and $n$ is a positive integer, then the number of elements required to topologically generate $G \times \mathbb{R}^{n}$ is exactly $n+1$. Note that the class of toplogical groups of this form is just the class of connected locally compact maximally almost periodic groups of weight at most $c$ [3].

\subsection{Definitions and notation}

(i) A topological group $G$ is said to be topologically finitely generated if there exists a positive integer $m$ such that $G=\overline{\operatorname{gp}\left\{x_{1}, x_{2}, \ldots, x_{m}\right\}}$, for some set $\left\{x_{1}, x_{2}, \ldots, x_{m}\right\}$ $\subseteq G$. If $n$ is the smallest such integer, then we say $\sigma(G)=n$.

(ii) Let $\left\{K_{j}: j \in J\right\}$ be the family of all compact connected simple simply-connected Lie groups. For each $j \in J$, let $C_{j}=\left(K_{j}\right)^{c}$, the product of $c$ copies of $K_{j}$, where $c$ is the cardinality of the continuum. Then we define

$$
\mathscr{C}=\prod_{j \in J} C_{j}
$$


As each $K_{j}$ is a simple Lie group, $\left(K_{j}\right)^{\prime}=K_{j}[2,5$ Corollaire de la Proposition 10]. So it follows that $\mathscr{C}$ is its own commutator subgroup; that is, $\overline{\mathscr{C}^{\prime}}=\mathscr{C}^{\prime}=\mathscr{C}$.

\section{Preliminary results}

The first five results are folklore, but we include some proofs for completeness.

Lemma 2.1. Let $A$ be a closed normal subgroup of a topological group $B$ and let $C$ be the quotient group $B / A$. If $A$ and $C$ are topologically finitely generated, then $B$ is topologically finitely generated and $\sigma(B) \leqq \sigma(A)+\sigma(C)$.

Lemma 2.2. Let $G$ and $H$ be topological groups. If $G$ is topologically finitely generated and there exists a continuous homomorphism of $G$ onto $H$, then $H$ is topologically finitely generated and $\sigma(G) \geqq \sigma(H)$.

The following proposition can be considered an interpretation of Kronecker's approximation theorem, [6, Theorem 28].

Proposition 2.3. If $n$ is a positive integer, then $\sigma\left(\mathbb{R}^{n}\right)=n+1$.

Proof. We have the following exact sequence:

$$
0 \rightarrow \mathbb{Z}^{n} \rightarrow \mathbb{R}^{n} \rightarrow \mathbb{R}^{n} / \mathbb{Z}^{n}=\mathbb{T}^{n} \rightarrow 0 .
$$

But $\sigma\left(\mathbb{Z}^{n}\right)=n$ and $\sigma\left(\mathbb{T}^{n}\right)=1[4, \S 25.14]$, and so by Lemma $2.1, \sigma\left(\mathbb{R}^{n}\right) \leqq n+1$.

Now suppose that $\sigma\left(\mathbb{R}^{n}\right)=m$ for some $m \leqq n$. Then there exists a set $B=\left\{b_{1}, b_{2}, \ldots, b_{m}\right\}$ $\subseteq \mathbb{R}^{n}$ such that $\mathbb{R}^{n}=\overline{g p\{B\}}$.

If $B$ is a linearly dependent set and $\operatorname{sp}\{B\}$ the vector subspace spanned by $B$, then

$$
\begin{aligned}
\overline{\operatorname{gp}\{B\}} & \subseteq \overline{\operatorname{sp}\{B\}} \\
& =\overline{\operatorname{sp}\left\{b_{1}, b_{2}, \ldots, b_{k}\right\}}, \text { for some } k<m \leqq n \\
& =\mathbb{R}^{k}, \text { as all vector subspaces of } \mathbb{R}^{n} \text { are closed. }
\end{aligned}
$$

Therefore $\overline{\operatorname{gp}\{B\}} \neq \mathbb{R}^{n}$.

If $B$ is linearly independent set, then, from Proposition 21 of $[6], \operatorname{gp}\{B\}$ is topologically isomorphic to $\mathbb{Z}^{m}$, and again we have $\overline{\operatorname{gp}\{B\}} \neq \mathbb{P}^{n}$.

Therefore $\sigma\left(\mathbb{R}^{n}\right) \neq m$ for any $m \leqq n$, and so $\sigma\left(\mathbb{R}^{n}\right)=n+1$.

Proposition 2.4. Let $K$ be a compact connected Hausdorff abelian group of weight $w(K) \leqq c$, and $n$ a non-negative integer. Then $\sigma\left(K \times \mathbb{R}^{n}\right)=n+1$.

Proof. Consider the exact sequence 


$$
0 \rightarrow \mathbb{Z}^{n} \rightarrow K \times \mathbb{R}^{n} \rightarrow K \times \mathbb{V}^{n} \rightarrow 0
$$

As $K \times \mathbb{T}^{n}$ is a compact connected Hausdorff abelian group with weight $w\left(K \times \mathbb{T}^{n}\right) \leqq c$, $[4, \S 25.14]$ implies that $\sigma\left(K \times \mathbb{V}^{n}\right)=1$. So by Lemma 2.1, we obtain $K \times \mathbb{R}^{n}$ is topologically finitely generated and $\sigma\left(K \times \mathbb{R}^{n}\right) \leqq \sigma\left(\mathbb{Z}^{n}\right)+\sigma\left(K \times \mathbb{T}^{n}\right)$. But, $\sigma\left(\mathbb{Z}^{n}\right)=n$ and so $\sigma\left(K \times \mathbb{R}^{n}\right) \leqq n+1$.

But as $\mathbb{R}^{n}$ is a quotient of $K \times \mathbb{R}^{n}, \sigma\left(K \times \mathbb{R}^{n}\right) \geqq \sigma\left(\mathbb{R}^{n}\right)=n+1$, from Proposition 2.3. Hence $\sigma\left(K \times \mathbb{R}^{n}\right)=n+1$.

Lemma 2.5. ([1, §9.1.1]) Let $A$ and $B$ be subsets of a topological group $G$. Then $\overline{[A, \bar{B}]}=\overline{[\bar{A}, \bar{B}]}$.

Theorem 2.6. ([5, Theorem 4.13]) If $G$ is a compact connected Hausdorff non-abelian topological group of weight $w(G) \leqq c$, then $\sigma(G)=2$.

Lemma 2.7. Let $A$ and $B$ be subgroups of a group $G$. Then $A$ and $B$ are contained in the normalizer of $[A, B]$. Further, if $G$ is generated by $A \cup B$ and $A$ and $B$ are abelian, then $G^{\prime}=[A, B]$.

Proof. That the normalizer of $[A, B]$ contains $A$ and $B$ follows from the identity

$$
[x y, z]=[y, z]^{x^{-1}}[x, z] .
$$

If $G$ is generated by $A \cup B$ then it follows that $[A, B]$ is a normal subgroup of $G$. If $A$ and $B$ are abelian, then obviously $G /[A, B]$ is abelian. Hence $G^{\prime} \subseteq[A, B]$. As $G^{\prime} \supseteq[A, B]$ trivially, we deduce that $G^{\prime}=[A, B]$.

Proposition 2.8. Let $G$ be a topological group topologically generated by two elements, $a$ and $b$. Then $\overline{G^{\prime}}=\overline{[\operatorname{gp}\{a\}, \operatorname{gp}\{b\}] \text {. }}$

Proof. From Lemma 2.7, we have $\overline{[\operatorname{lgp}\{a\}, \operatorname{gp}\{b\}]}=\overline{(\operatorname{gp}\{a, b\})^{\prime}}$, so

$$
\begin{aligned}
\overline{G^{\prime}} & =\overline{[\overline{\operatorname{gp}\{a, b\}}, \overline{\operatorname{gp}\{a, b\}}}, \\
& =\overline{[\operatorname{gp}\{a, b\}, \overline{\operatorname{gp}\{a, b\}]}}, \text { from Lemma } 2.5 \\
& =\overline{(\operatorname{gp}\{a, b\})^{\prime}} \\
& =\overline{[\operatorname{gp}\{a\}, \operatorname{gp}\{b\}]} .
\end{aligned}
$$

\section{The main result}

The following result is the key to Theorem 3.2 , the main result of this paper. 
Theorem 3.1. Let $G$ be any compact connected Hausdorff group of weight $w(G) \leqq c$. Then there exists a compact connected Hausdorff abelian group $K$ of weight $w(K) \leqq c$ and a continuous homomorphism from $K \times \mathscr{C}$ onto $G$.

Proof. There exists a family of compact connected simple simply-connected Lie groups $\left\{G_{i}: i \in I\right\}$, a compact connected abelian subgroup $K$ of $G$, and a continuous homomorphism $\phi$ of $\prod_{i \in I} G_{i} \times K$ onto $G$, with $\operatorname{ker} \phi$ a totally disconnected subgroup [7, Theorem 6.5.6]. Note that $w(K) \leqq w(G) \leqq c$.

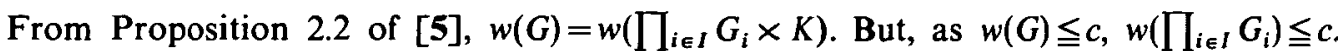
So $\max \left\{\right.$ card $\left.l, w\left(G_{i}\right), w(K)\right\} \leqq c$ and therefore card $l \leqq c$.

Note that the family of all compact connected simple simply-connected Lie groups in countable. It follows from this, and the fact that card $I \leqq c$, that there is a continuous homomorphism of $\mathscr{C}$ into $\prod_{i \in I} G_{i}$. Therefore there is a continuous homomorphism $\psi$ of $\mathscr{C} \times K$ onto $\prod_{i \in I} G_{i} \times K$.

Finally, the map $\theta=\phi \circ \psi$ is the required continuous homomorphism of $\mathscr{C} \times K$ onto G.

Theorem 3.2. Let $G$ be any compact connected Hausdorff group with weight $w(G) \leqq c$, and $n$ a positive integer. Then $\sigma\left(G \times \mathbb{R}^{n}\right)=n+1$.

Proof. If $G$ is abelian, then from Proposition $2.4, \sigma\left(G \times \mathbb{R}^{n}\right)=n+1$. Now assume that $G$ is non-abelian.

From Theorem $3.1, G \times \mathbb{R}^{n}$ is a quotient group of $\mathscr{C} \times K \times \mathbb{R}^{n}$ for some compact connected Hausdorff abelian group $K$ of weight $w(K) \leqq c$. We will show that $\mathscr{C} \times K \times \mathbb{B}^{n}$ can be topologically generated by $n+1$ elements. From this we can deduce that $G \times \mathbb{R}^{n}$ can be topologically generated by $n+1$ elements. As $\mathbb{R}^{n}$ is a quotient group of $G \times \mathbb{R}^{n}$, using Proposition 2.3 we will then see that $\sigma\left(G \times \mathbb{R}^{n}\right) \geqq \sigma\left(\mathbb{R}^{n}\right)=n+1$. This will complete the proof.

As $\mathscr{C}$ is a compact connected Hausdorff group with $w(\mathscr{C}) \leqq c$, Theorem 2.6 implies that $\sigma(\mathscr{C})=2$. Let $\{a, b\}$ topologically generate $\mathscr{C}$,

From Proposition 2.4, we have $\sigma\left(K \times \mathbb{R}^{n}\right)=n+1$. Let $\left\{f_{1}, f_{2}, \ldots, f_{n+1}\right\}$ be a set of topological generators for $K \times \mathbb{R}^{n}$.

We shall show that the set $S=\left\{\left(a, f_{1}\right),\left(b, f_{2}\right),\left(b, f_{3}\right), \ldots,\left(b, f_{n+1}\right)\right\}$ topologically generates $\mathscr{C} \times K \times \mathbb{R}^{n}$.

We prove that $H=\operatorname{gp}\left\{\left(a^{-p} b^{-q} a^{p} b^{q}, e\right): p, q \in \mathbb{Z}\right\} \subseteq \operatorname{gp}\{S\}$, where $e$ is the identity of the group. To do this, it is enough to show that for any $p, q \in \mathbb{Z},\left(a^{-p} b^{-q} a^{p} b^{q}, e\right) \in \operatorname{gp}\{S\}$. Let $p, q \in \mathbb{Z}$. As $K$ is abelian, for any $i \in\{2,3, \ldots, n+1\}$,

$$
\begin{aligned}
\left(a^{-p} b^{-q} a^{p} b^{q}, e\right) & \left.=\left(a^{-p}, f_{1}^{-p}\right)\left(b^{-q}, f_{i}^{-q}\right)\left(a^{p}, f_{1}^{p}\right) b^{q}, f_{i}^{q}\right) \\
& =\left(a, f_{1}\right)^{-p}\left(b, f_{i}\right)^{-q}\left(a, f_{1}\right)^{p}\left(b, f_{i}\right)^{q} \\
& \in \operatorname{gp}\{S\} .
\end{aligned}
$$

By Proposition 2.8, this implies that $\overline{\mathscr{C}^{\prime} \times\{\boldsymbol{e}\}}=\bar{H} \subseteq \overline{\mathrm{gp}\{S\}}$. But, as observed in 1.2(ii), $\mathscr{C}^{\prime}=\mathscr{C}$. So $\mathscr{C} \times\{e\} \subseteq \overline{\mathrm{gp}\{S\}}$. 
As $\left(a^{-1}, e\right) \in \mathscr{C} \times\{e\}$ and $\left(a, f_{1}\right) \in \operatorname{gp}\{S\}$, it follows that $\left(a^{-1}, e\right)\left(a, f_{1}\right)=\left(e, f_{1}\right) \in \overline{\operatorname{gp}}\{\bar{S}\}$. Similarly, $\left(e, f_{i}\right) \in \overline{\operatorname{gp}\{S\}}$ for $i \in\{2,3, \ldots, n+1\}$.

We now have $\{e\} \times\left(K \times \mathbb{R}^{n}\right) \subseteq \overline{\operatorname{gp}\{S\}}$ and $\mathscr{C} \times\{e\} \subseteq \overline{\operatorname{gp}\{S\}}$, and so $\mathscr{C} \times\left(K \times \mathbb{R}^{n}\right) \subseteq \overline{\operatorname{gp}\{S\}}$. Therefore $\mathscr{C} \times\left(K \times \mathbb{R}^{n}\right)=\overline{\operatorname{gp}\{S\}}$, as required.

Hence $\sigma\left(\mathscr{C} \times K \times \mathbb{R}^{n}\right) \leqq n+1$, and the result follows.

Corollary. Let $L$ be a connected locally compact Hausdorff abelian group of weight $w(L) \leqq c$, and $G$ a compact connected Hausdorff group of weight $w(G) \leqq c$. If $L$ is compact and $G$ is abelian, then $\sigma(G \times L)=1$. If $L$ is compact and $G$ is non-abelian, then $\sigma(G \times L)=2$. If $L$ is non-compact, then it is topologically isomorphic to $\mathbb{R}^{n} \times K$ for $n$ a positive integer and $K$ a compact connected Hausdorff group, and $\sigma(G \times L)=n+1$.

Proof. If $L$ is compact and $G$ is abelian, then $G \times L$ is a compact connected Hausdorff abelian group, and so from [4, § 25.14], $\sigma(G \times L)=1$.

If $L$ is compact and $G$ is non-abelian, then $G \times L$ is a compact connected Hausdorff non-abelian group. Therefore, from Theorem 2.6, $\sigma(G \times L)=2$.

If $L$ is non-compact, then $L$ is topologically isomorphic to $K \times \mathbb{R}^{n}$ where $n \geqq 1$ and $K$ is a compact connected Hausdorff abelian group of weight $w(K) \leqq c$.

By Theorem 3.1, $G \times L$ is a quotient group of $\mathscr{C} \times K_{1} \times L$, for some compact connected Hausdorff abelian group $K_{1}$ with weight $w\left(K_{1}\right) \leqq c$. So $G \times L$ is a quotient group of $\mathscr{C} \times K_{1} \times K \times \mathbb{R}^{n}$. But by Theorem 3.2, $\sigma\left(\mathscr{C} \times K_{1} \times K \times \mathbb{R}^{n}\right)=n+1$ and so $\sigma(G \times L) \leqq n+1$.

As $L$ is a quotient of $G \times L$, Lemma 2.2 implies that $\sigma(G \times L) \geqq \sigma(L)$. But from Proposition $2.4, \sigma(L)=\sigma\left(\mathbb{R}^{n} \times K\right)=n+1$, and therefore, $\sigma(G \times L) \geqq n+1$.

Hence $\sigma(G \times L)=n+1$.

\section{REFERENCES}

1. N. Bourbakı, Lie Groups and Lie Algebras, Chapters 1-3 (Springer-Verlag, Berlin, 1989).

2. N. Bourbakı, Groupes et algèbres de Lie, Chap. IX, Groupes de Lie rèels compacts (Masson, Paris, 1982).

3. S. Grosser and M. Moskowitz. Compactness conditions in topological groups, J. Reine Angew. Math. 246 (1971), 1-40.

4. E. Hewitr and K. A. Ross, Abstract Harmonic Analysis I (Springer-Verlag, Berlin, 1963).

5. K. H. Hofmann and S. A. Morris, Weight and c, J. Pure Appl. Algebra 68 (1990), 181-194.

6. S. A. Morris, Pontryagin Duality and the Structure of Locally Compact Abelian Groups (Cambridge University Press, Cambridge, 1977).

7. J. F. Price, Lie Groups and Compact Groups (Cambridge University Press, Cambridge, 1977).

Dublin Institute of Technology

Kevin ST.

DUBLIN, 8

IRELAND
UNIVERSITY OF WOLLONGONG

Wollongong

N.S.W. 2522,

Australia 